\title{
The Role of Wave-Induced Coriolis-Stokes Forcing on the Wind-Driven Mixed Layer
}

\author{
Jeff A. Polton, ${ }^{*}$ David M. Lewis, ${ }^{+}$and Stephen E. Belcher \\ Department of Meteorology, University of Reading, Reading, Berkshire, United Kingdom
}

(Manuscript received 11 July 2003, in final form 27 August 2004)

\begin{abstract}
The interaction between the Coriolis force and the Stokes drift associated with ocean surface waves leads to a vertical transport of momentum, which can be expressed as a force on the mean momentum equation in the direction along wave crests. How this Coriolis-Stokes forcing affects the mean current profile in a wind-driven mixed layer is investigated using simple models, results from large-eddy simulations, and observational data. The effects of the Coriolis-Stokes forcing on the mean current profile are examined by reappraising analytical solutions to the Ekman model that include the Coriolis-Stokes forcing. Turbulent momentum transfer is modeled using an eddy-viscosity model, first with a constant viscosity and second with a linearly varying eddy viscosity. Although the Coriolis-Stokes forcing penetrates only a small fraction of the depth of the wind-driven layer for parameter values typical of the ocean, the analytical solutions show how the current profile is substantially changed through the whole depth of the wind-driven layer. It is shown how, for this oceanic regime, the Coriolis-Stokes forcing supports a fraction of the applied wind stress, changing the boundary condition on the wind-driven component of the flow and hence changing the current profile through all depths. The analytical solution with the linearly varying eddy viscosity is shown to reproduce reasonably well the effects of the Coriolis-Stokes forcing on the current profile computed from large-eddy simulations, which resolve the three-dimensional overturning motions associated with the turbulent Langmuir circulations in the wind-driven layer. Last, the analytical solution with the CoriolisStokes forcing is shown to agree reasonably well with current profiles from previously published observational data and certainly agrees better than the standard Ekman model. This finding provides evidence that the Coriolis-Stokes forcing is an important mechanism in controlling the dynamics of the upper ocean.
\end{abstract}

\section{Introduction}

The oceanic wind-driven current profile is difficult to observe because the velocities are small and of similar magnitude to the velocities associated with inertial oscillations and surface wave motions. Consequently there are few detailed measurements of the Ekman current profile. Observations suggest three features of the wind-driven current profile that need to be explained by any model. First, the surface current lies at an angle of between $10^{\circ}$ and $45^{\circ}$ to the surface wind stress (Huang 1979). Second, at a depth between 5 and $20 \mathrm{~m}$ the current is deflected by approximately $75^{\circ}$ to the

\footnotetext{
* Current affiliation: Scripps Institution of Oceanography, University of California, San Diego, La Jolla, California.

+ Current affiliation: Department of Mathematics, University of Liverpool, Liverpool, United Kingdom.
}

Corresponding author address: S. E. Belcher, Department of Meteorology, University of Reading, P.O. Box 243, Reading, Berkshire RG6 6BB, United Kingdom.

E-mail: s.e.belcher@reading.ac.uk wind stress (Price and Sundermeyer 1999). Third, the current speed is rapidly attenuated with depth. The classical Ekman model cannot explain all these observed features (Lewis and Belcher 2004).

The difficulties in observation due to similarity in magnitude between the current speed and the speeds associated with other physical processes suggest that other processes may be dynamically important. Surface waves are a ubiquitous feature of the ocean surface. The leading order water motions associated with the surface waves are periodic and, at least below the troughs, do not affect the time-averaged, mean, current profile. Surface waves also produce a mean Lagrangian transport in their direction of propagation, the Stokes drift (e.g., Phillips 1977). For a monochromatic wave the Stokes drift is given by

$$
u_{s}=U_{s} e^{2 k z} \text { and } U_{s}=(a k)^{2} c,
$$

for wave amplitude $a$, wavenumber $k$, wave phase speed $c$, and depth $z$ that is zero at the mean sea level and decreasing downward. The significance is that, in an inviscid fluid, lines of vorticity move with fluid parcels, and so the Stokes drift tilts and stretches initially 
vertical vorticity into the horizontal plane. In the ocean mixed layer there are two sources of vertical vorticity: vorticity from three-dimensional turbulent motions within the mixed layer and planetary vorticity. Distortion of turbulent vorticity by Stokes drift is at the heart of models for Langmuir circulations (Leibovich 1983; McWilliams et al. 1997; Teixeira and Belcher 2002). The interaction of the Stokes drift with planetary vorticity is the subject of this paper.

The effects of Stokes drift in a rotating frame was first considered by Ursell (1950), Hasselmann (1970), and Pollard (1970) who showed that, for an inviscid ocean, there can be no net mass transport associated with the Stokes drift. Subsequently, also using a Lagrangian description, Weber (1983a, b) showed how including viscosity, no matter how small, yields a nonzero net mass transport. Hasselmann (1970) also shows that the interaction between the planetary vorticity and the Stokes drift yields a force on the Eulerian momentum balance: $\mathbf{f} \times \mathbf{u}_{s}$. To give expression to this process, here we refer to this forcing as the Coriolis-Stokes forcing. The origin of this forcing can be understood in two ways. First, and intuitively, the Stokes drift attempts to tilt and stretch the planetary vorticity into the horizontal leading to a vortex force on the flow. Alternatively, the $\mathbf{f} \times \mathbf{u}_{s}$ forcing can be interpreted as the divergence of a wave-induced stress as follows. The Eulerian velocity is decomposed into a rapidly varying wave orbital velocity, $\tilde{u}$, and a mean velocity, $\bar{u}$. On a rotating ocean, the plane of the orbital velocity is tilted in the along wave crest direction by the Coriolis acceleration, as shown schematically in Fig. 1. This tilting introduces an along wave crest component into the orbital velocity, $\tilde{v}$, which is correlated with the vertical component, $\tilde{w}$. Hence there is a wave-induced stress $\rho \overline{\tilde{v} \tilde{w}}$, whose divergence Hasselmann (1970) shows can be expressed as

$$
-\rho \frac{\partial}{\partial z} \overline{\tilde{v} \tilde{w}}=-\rho\left|\mathbf{f} \times \mathbf{u}_{s}\right|,
$$

acting in the direction along wave crests. It is the effect of this Coriolis-Stokes forcing on vertical profiles of the mean current that is the focus of this paper.

When the momentum equations are averaged over the wave periods, the Coriolis-Stokes forcing appears as an extra term in the mean horizontal momentum equations that govern the ageostrophic motions so that

$$
\rho f \hat{\mathbf{z}} \times\left(\mathbf{u}+\mathbf{u}_{s}\right)=\frac{\partial \boldsymbol{\tau}}{\partial z},
$$

where $\rho$ is the density and $\tau$ is the turbulent stress (see Huang 1979). The boundary conditions are a constant wind stress, $\tau_{0}$, at the mean sea surface $z=0$ and the turbulent stress and ageostrophic velocity tend to zero at large depth:

$$
\tau_{0}=\rho v_{*}^{2} \hat{\mathbf{x}} \text { on } z=0 ; \quad \boldsymbol{u} \rightarrow 0, \quad \boldsymbol{\tau} \rightarrow 0 \text { as } z \rightarrow-\infty .
$$

Here $v_{*}$ is the friction velocity in the water. There are two important depth scales in this problem. First, there is the Stokes depth scale, $\delta_{s}=1 /(2 k)$, over which the Stokes drift, $\mathbf{u}_{s}$, and the $\mathbf{f} \times \mathbf{u}_{s}$ forcing penetrate. Second, there is the Ekman depth scale, $\delta_{e}$, over which turbulent stresses balance Coriolis force. Typical values for these parameters in the open ocean mixed layer are $\delta_{e} \approx 50 \mathrm{~m}$, which is much greater than $\delta_{s} \approx 5 \mathrm{~m}$.

\section{a. Magnitude of the Coriolis-Stokes forcing}

What is the magnitude of the Coriolis-Stokes forcing in comparison with the terms in the standard Ekman balance? The depth-integrated transports give a first indication; they are defined by

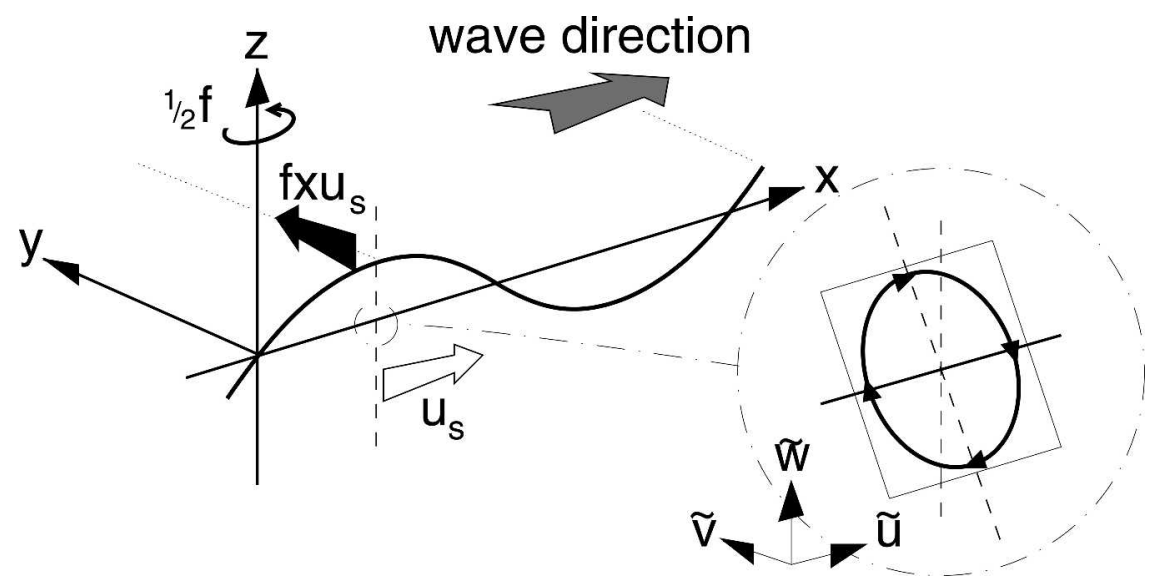

FIG. 1. Schematic illustrating the orbital path for a particle under a wave is tilted, by planetary rotation, in the along-wave crest direction. The new $\tilde{v}$ component orbital velocity correlates with the $\tilde{w}$ component to produce a nonzero stress. The divergence of this stress can be written as (Hasselmann 1970) $-\rho \mathbf{f} \times \mathbf{u}_{s}$. 


$$
\mathbf{T}=\int_{-\infty}^{0} \mathbf{u} d z=\mathbf{U}_{\boldsymbol{e}} \delta_{e} \quad \text { and } \quad \mathbf{T}_{s}=\int_{-\infty}^{0} \mathbf{u}_{s} d z=\mathbf{U}_{\boldsymbol{s}} \delta_{s} .
$$

Integration of (3) and rearranging gives

$$
\mathbf{T}+\mathbf{T}_{s}=-\frac{\hat{\mathbf{z}} \times \boldsymbol{\tau}_{\mathbf{0}}}{f \rho},
$$

which shows that the net (Eulerian plus Lagrangian) transport is unaffected by the waves (see McWilliams and Restrepo 1999). We define the Ekman-Stokes number, $E_{s}$ (cf. McWilliams and Restrepo 1999), to be a measure of the wave-induced transport in comparison with the total net transport, namely

$E_{s}=\frac{\text { wave-induced transport }}{\text { total net transport }}=\frac{\left|\mathbf{T}_{s}\right|}{\left|\hat{\mathbf{z}} \times \tau_{\mathbf{0}} \rho f\right|}=\frac{U_{s} \delta_{s}}{U_{e} \delta_{e}}$,

where $U_{e}$ is the velocity scaling for the pure Ekman current, defined by the transport relation (5), such that $U_{e} \delta_{e}=v_{*}^{2} / f$. We evaluate this expression for $E_{s}$ by using the formula for $u_{s}$ as a function of wind speed at $19.5 \mathrm{~m}$ obtained by Kenyon (1969) based on the Pierson and Moskowitz (1964) spectrum of the fully developed sea, which is then integrated over depth to give the wavedriven transport. Assuming a log profile for the wind speed, $E_{s}$ is then a function of $U_{10}$ given by

$$
E_{s}=0.39 \frac{f U_{10}}{c_{D}}\left(1+\frac{c_{D}^{1 / 2} \ln 1.95}{\kappa}\right)^{3},
$$

where $c_{D}$ is the atmospheric drag coefficient defined by $\tau_{0}=\rho_{a} c_{D} U_{10}^{2}$, with air density $\rho_{a}=1.2 \mathrm{~kg} \mathrm{~m}^{-3}$ and $c_{D}$ taken from Garratt [1992, Eq. (4.24)], namely $c_{D}=$ $\left(0.75+0.067 U_{10}\right) \times 10^{-3}$.

Figure 2 shows how $E_{s}$ increases with wind speed at four latitudes. For example, at a latitude of $50^{\circ} \mathrm{N}$, $E_{s}\left(U_{10}=12 \mathrm{~m} \mathrm{~s}^{-1}\right)=0.4$ suggesting that the wave-

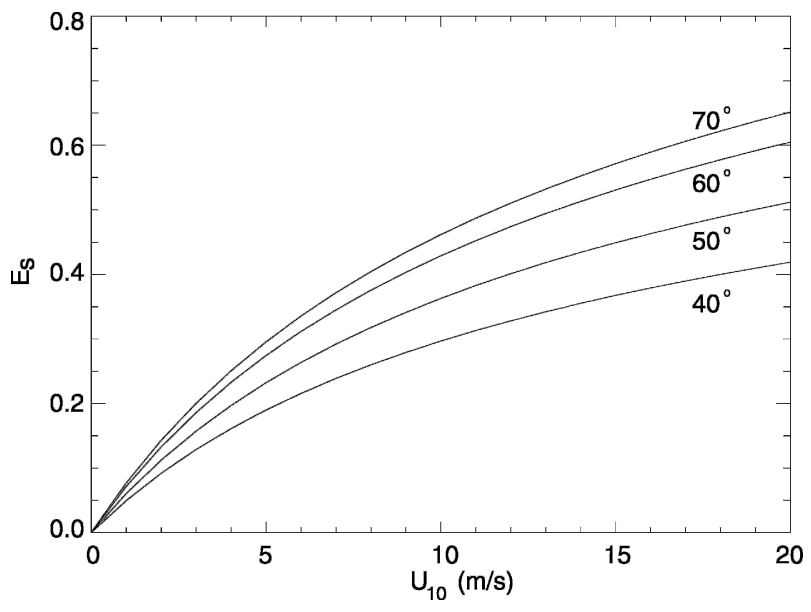

FIG. 2. Graph showing how $E_{s}$, the ratio of wave-induced to total net transport, varies with $U_{10}$ for four different latitudes: $40^{\circ}$, $50^{\circ}, 60^{\circ}$, and $70^{\circ}$. Even moderate wind speeds of $U_{10}=12 \mathrm{~m} \mathrm{~s}^{-1}$ suggest that wave-induced effects could have a nonnegligible impact in the ocean mass transport. induced transport can be a significant fraction of the wind-induced transport. These plots can be compared with those in McWilliams and Restrepo (1999) who deduce global maps of $E_{s}$ based on climatological winds (although they appear to use the $19.5-\mathrm{m}$ wind speed in place of $U_{10}$ ). The finding that the Eulerian depthintegrated wave-driven transport is comparable to the wind-forced Ekman transport motivates the present analysis, which aims to determine the vertical distribution of the Eulerian wave-driven transport in the Ekman-Stokes layer.

\section{b. Aims of the present paper}

Madsen (1978) and Huang (1979) showed that the Coriolis-Stokes forcing acts in combination with the Coriolis force and the divergence of vertical momentum transfer by turbulent stresses, thereby changing the usual Ekman balance in the wind-driven mixed layer and the current profiles. Later studies have developed the theory for more sophisticated representations of the turbulent stress (Jenkins 1986, 1987), for finite depths (Xu and Bowen 1994), and for the role of Langmuir circulations (Gnanadesikan and Weller 1995). A number of questions remain however.

Huang's (1979) solution shows that the CoriolisStokes forcing, which penetrates only into shallow depths, affects the current profiles through its whole depth. So, the first question is by what physical mechanism is the whole depth affected? Here we address this question in section 2 by reappraising Huang's analytical solution for the current profile. This reappraisal shows how the depth-integrated transport is partitioned between a portion that occupies only the Stokes depth and another portion that occupies the whole winddriven mixed layer. This analysis then shows how for practical purposes the Coriolis-Stokes forcing can be represented as an effective boundary condition on the standard Ekman flow.

A second question is what evidence is there that the role of the Coriolis-Stokes forcing is real and measurable? To address this question we examine effects of the Coriolis-Stokes forcing on the otherwise standard Ekman dynamics: we ignore further complicating effects of stratification, etc, to isolate the role of CoriolisStokes forcing. The question is addressed here in two ways. First, in section 3 the results of the simple models are compared with current profiles computed from large-eddy simulations of the wind-driven ocean mixed layer that account for the effects of the Stokes drift. And second, in section 4 the results of the simple model are compared with previous observations of the winddriven ocean mixed layer.

\section{Structure of the mean current profile in the Ekman-Stokes layer}

The Coriolis-Stokes forcing changes the dynamical balance in wind driven mixed layer and, hence, the 
structure of the mean current profile, resulting in the Ekman-Stokes layer. In this section we reappraise solutions to simple models to show how this forcing changes the currents over the whole depth of the layer. This analysis then suggests a practical method for incorporating the Coriolis-Stokes forcing into large-scale models.

\section{a. Current profile with a constant eddy viscosity}

Just as for the classical Ekman layer, many of the characteristics of the current in the Ekman-Stokes layer are shown in the solution to the dynamical equations with a constant eddy viscosity $\kappa_{m}$. This problem was first considered by Madsen (1978) and Huang (1979). Here we reappraise the solution by writing it in a form that highlights how the shallow wave process change the current profile over the whole depth of the Ekman-Stokes layer.

The turbulent stress in (3), $\boldsymbol{\tau}$, is parameterized with a simple gradient transfer eddy-viscosity model:

$$
\boldsymbol{\tau}=\rho \kappa_{m} \frac{\partial \mathbf{u}}{\partial z}
$$

where $\kappa_{m}$ is the eddy viscosity, taken in this subsection to be constant.

Huang (1979) shows that the solution is obtained by recasting the momentum equation [(3)] into complex notation, where $\mathbf{u}=u \mathbf{i}+v \mathbf{j}$ is re-expressed as $\mathcal{u}=u+$ $i v$. The solution to (3) is then obtained using standard methods. Here we decompose this solution to the Eulerian velocity into three parts, thus

$$
\mathcal{U}=\mathcal{U}_{e}+\mathcal{U}_{e s}+\mathcal{U}_{s},
$$

where

$$
\begin{aligned}
\mathcal{U}_{e}= & (1-i) U_{e} \exp \left[(1+i) \frac{z}{\delta_{e}}\right], \\
\mathcal{U}_{e s}= & (1-i) U_{e} \exp \left[(1+i) \frac{z}{\delta_{e}}\right] \\
& \times\left[\frac{U_{s} \delta_{s}}{U_{e} \delta_{e}} \frac{\frac{1}{2} \delta_{e}^{2} / \delta_{s}^{2}}{\left(1+i \frac{1}{2} \delta_{e}^{2} / \delta_{s}^{2}\right)}\right], \text { and } \\
\mathcal{U}_{s}= & -\frac{U_{s}}{\left(1+i \frac{1}{2} \delta_{e}^{2} / \delta_{s}^{2}\right)} \exp \left(\frac{z}{\delta_{s}}\right) .
\end{aligned}
$$

In this case, $\delta_{e}=\left(2 \kappa_{m} / f\right)^{1 / 2}$. Writing the solution in this way enables clear physical interpretation of the role of the Coriolis-Stokes forcing. Here $\mathcal{U}_{e}$ is the pure Ekman solution and would be the entire solution if the waveinduced affects were not included. However, the Stokes-Coriolis forcing introduces two new terms into the solution. First, there is a Stokes component of the current, $\mathcal{U}_{s}$. This part of the solution is forced directly by the Coriolis-Stokes force; mathematically it arises as a particular integral to the Coriolis-Stokes forcing. The Stokes component of the current decays over the Stokes depth scale, $\delta_{s}$. (The Stokes component of the Eulerian current $\mathcal{U}_{s}$ is the dynamical response to the Coriolis-Stokes forcing and should not be confused with the Lagrangian Stokes drift, $u_{s}$.) Second, there is an Ekman-Stokes component of the current, $\mathcal{U}_{e s}$. Important is that this term decays over the Ekman depth scale, $\delta_{e}$, and so changes the current profile through the whole depth of the layer. This part of the solution arises to ensure that the total solution satisfies the wind stress boundary condition imposed at the sea surface. That is, the Stokes component of the solution leads to a surface stress: the Ekman-Stokes component is required to remove this surface stress. Since the Ekman-Stokes has the same depth variation as the Ekman part of the solution, it is as if we have the Ekman solution but with a changed surface boundary condition. In this sense the effect of the Coriolis-Stokes forcing is to change the boundary condition on the Ekman current.

The decomposition of the solution (11)-(13) is shown in a hodograph and as depth profiles in Fig. 3. The thick lines represent the full solution, the thin solid lines represent the Ekman component, the dashed lines denote the Ekman-Stokes component, and the dotted-dashed lines denote the Stokes current component. Notice how the Ekman-Stokes component of the solution penetrates through the whole depth of the wind-driven layer, whereas the Stokes component of the solution penetrates only the upper fraction of the layer. The wave-induced effect is to further rotate the current vectors, as compared with the pure Ekman solution.

The analysis of section 1a showed the magnitude of the depth integrated wave-driven transport. Now we can understand the depth profile of this transport. Consider then the depth-integrated Eulerian transport carried by each of the three components of the solution in (10):

$$
\begin{aligned}
& \mathcal{T}_{e}=-i U_{e} \delta_{e}, \quad \mathcal{T}_{e s}=-U_{s} \delta_{s} \frac{\frac{1}{2} i \delta_{e}^{2} / \delta_{s}^{2}}{1+\frac{1}{2} i \delta_{e}^{2} / \delta_{s}^{2}}, \text { and } \\
& \mathcal{T}_{s}=-U_{s} \delta_{s} \frac{1}{1+\frac{1}{2} i \delta_{e}^{2} / \delta_{s}^{2}} .
\end{aligned}
$$

If the positive real axis is taken to be east, with wind and waves in that direction, then the depth-integrated wind-driven transport, $\mathcal{T}_{e}$, equals the value obtained in section 1a and is to the south. The sum of the transports arising from the Coriolis-Stokes forcing yield the total wave-driven transport-that is, $\mathcal{T}_{e s}+\mathcal{T}_{s}=-U_{s} \delta_{s}$. The power of the decomposition in (10) is now clear: the total wave-driven Eulerian transport is partitioned be- 

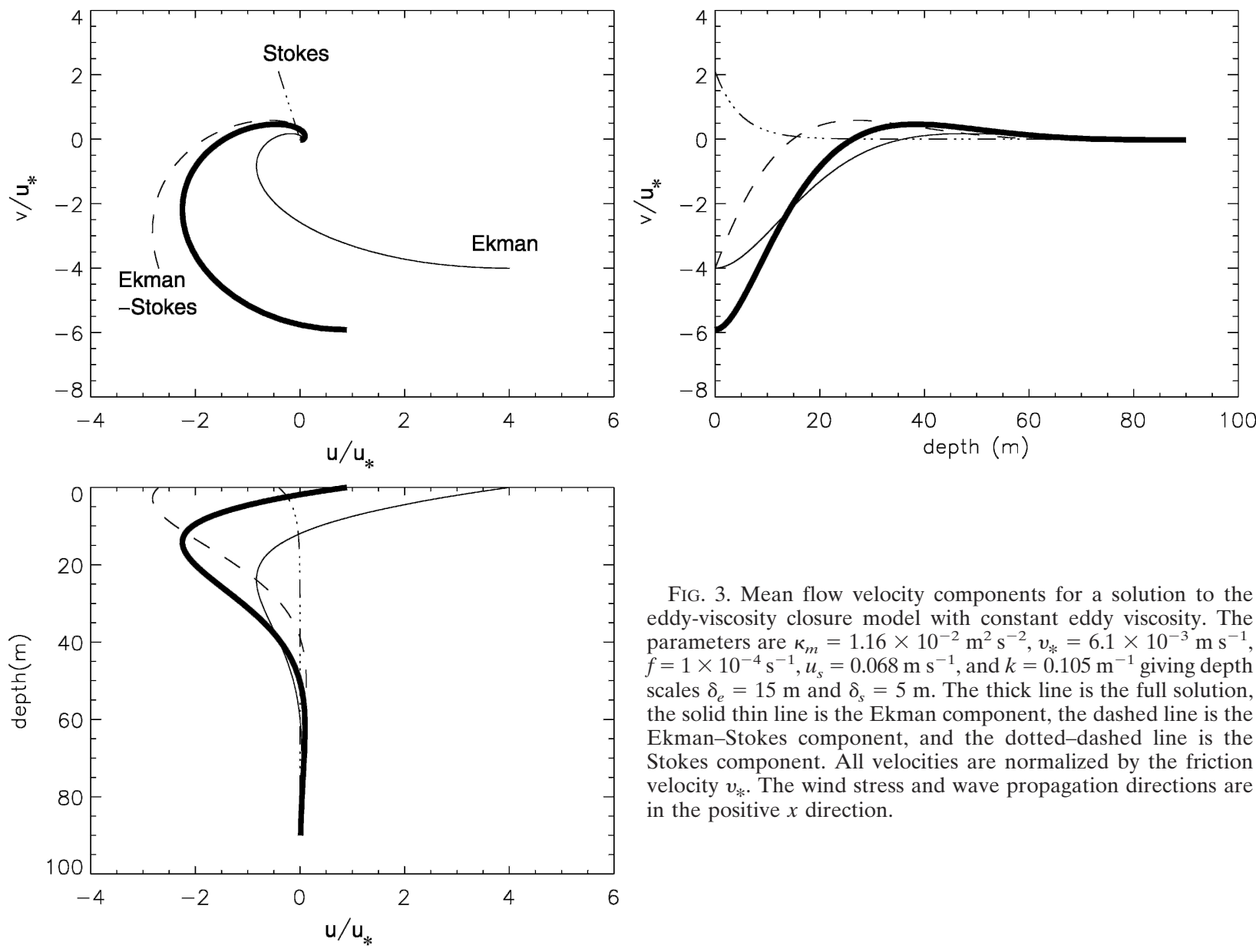

FIG. 3. Mean flow velocity components for a solution to the eddy-viscosity closure model with constant eddy viscosity. The parameters are $\kappa_{m}=1.16 \times 10^{-2} \mathrm{~m}^{2} \mathrm{~s}^{-2}, v_{*}=6.1 \times 10^{-3} \mathrm{~m} \mathrm{~s}^{-1}$, $f=1 \times 10^{-4} \mathrm{~s}^{-1}, u_{s}=0.068 \mathrm{~m} \mathrm{~s}^{-1}$, and $k=0.105 \mathrm{~m}^{-1}$ giving depth scales $\delta_{e}=15 \mathrm{~m}$ and $\delta_{s}=5 \mathrm{~m}$. The thick line is the full solution, the solid thin line is the Ekman component, the dashed line is the Ekman-Stokes component, and the dotted-dashed line is the Stokes component. All velocities are normalized by the friction velocity $v_{*}$. The wind stress and wave propagation directions are in the positive $x$ direction.

tween $\mathcal{T}_{e s}$, which extends through depth $\delta_{e}$, and $\mathcal{T}_{s}$, which extends through depth $\delta_{s}$. Figure 4 shows how these vector transports vary with the controlling parameter, $\delta_{e} / \delta_{s}$. With the wind stress to the east, the total wave-driven Eulerian transport, $\mathcal{T}_{e s}+\mathcal{T}_{s}$, is to the west. As shown in Fig. 4, the vector transports $\mathcal{T}_{\text {es }}$ and $\mathcal{T}_{s}$ lie on opposite sides of a circle with a diameter equal to the total Eulerian wave-driven transport. The vector $\mathcal{T}_{s}$ makes an angle $\theta$, given by $\tan \theta=1 / 2 \delta_{e}^{2} / \delta_{s}^{2}$, to the west; $\mathcal{T}_{s}$ has a northerly component, and $\mathcal{T}_{\text {es }}$ has a southerly component.

Hence the net water transport, which is the sum of the Eulerian and Lagrangian transports, has a complicated structure. Over the shallow Stokes depth, the net water transport is the sum of the Eulerian transport $\mathcal{T}_{s}$ and the depth-integrated Lagrangian Stokes drift $\mathcal{T}_{s}^{L}=U_{s} \delta_{s}$. The resultant $\mathcal{T}_{s}+\mathcal{T}_{s}^{L}=-\mathcal{T}_{\text {es }}$ has a northerly component, as shown in Fig. 4. Over the depth of the wind-driven layer, the net water transport is the sum of the usual southward wind-driven Ekman transport, $\mathcal{T}_{e}$, and the wave-driven transport, $\mathcal{T}_{e s}$. The resultant, $\mathcal{T}_{\text {es }}+\mathcal{T}_{e}$, has a westward component as shown in Fig. 4.

When $\delta_{e} / \delta_{s}$ is small, $\theta$ becomes small and the wave- driven transport is carried by $\mathcal{T}_{s}$ over the Stokes depth. This is the limit of no wind when the wave-driven Eulerian transport is equal and opposite to the Lagrangian Stokes drift at every depth. When $\delta_{e} / \delta_{s}$ is large, which is typical of the open ocean, most of the wave-driven transport is carried by $\mathcal{T}_{\text {es }}$ over the whole depth of the wind-driven mixed layer. In that case and for depths greater than the Stokes depth, $|z| \gg \delta_{s}$, the solution for the velocity becomes approximately the sum of (11) and (12) and can be written

$$
\mathcal{U} \approx\left(1-i E_{s}\right) \mathcal{U}_{e}
$$

where $E_{s}=U_{s} \delta_{s} / U_{e} \delta_{s}$. The Coriolis-Stokes forcing adds a new component to the standard Ekman solution that accounts for the depth-integrated Eulerian transport by the waves and has the same variation with depth as the standard Ekman solution.

We shall see in section $2 \mathrm{c}$ below that this new appraisal of Huang's solution demonstrates an efficient way of representing the Coriolis-Stokes forcing in large-scale ocean models. First we analyze a more faithful model for the turbulent stress. 


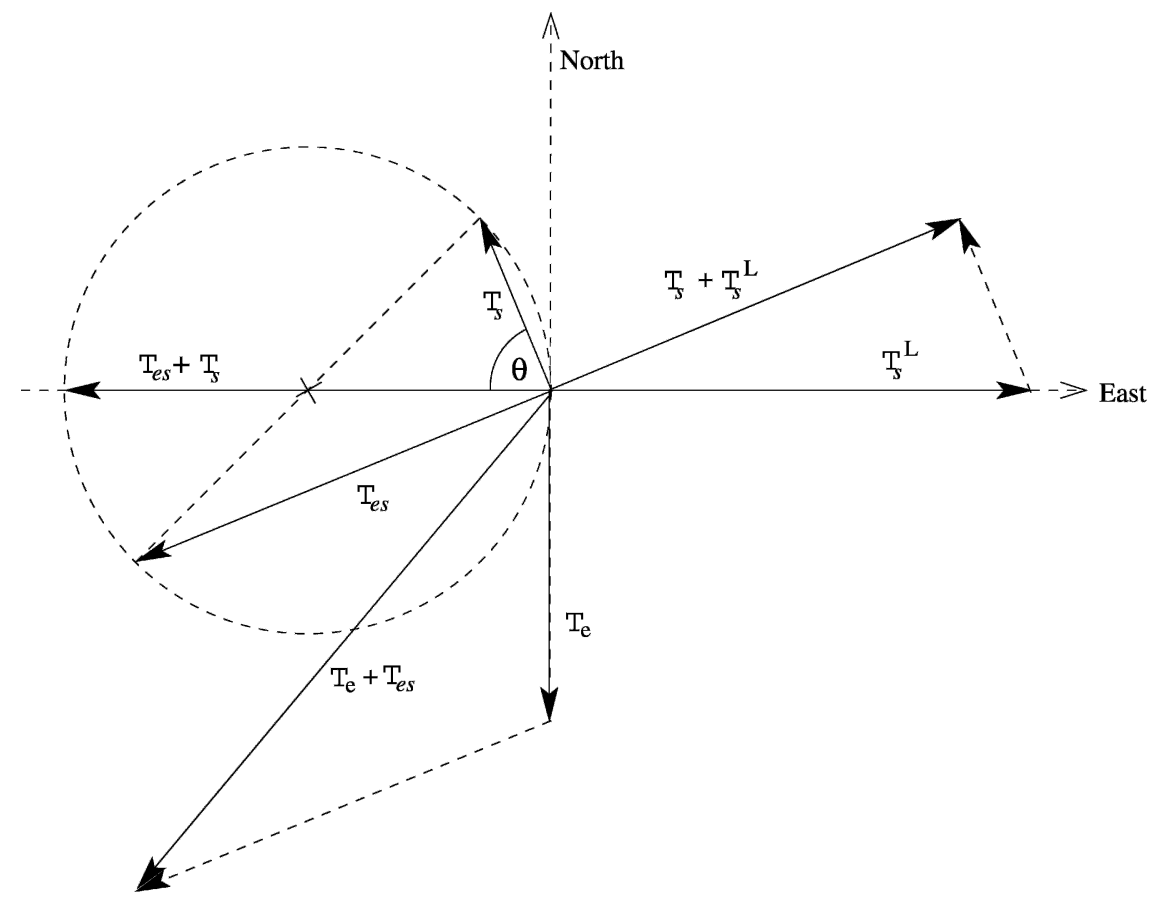

FIG. 4. Schematic showing the components of the vector depth-integrated transport. The Eulerian wave-driven transport has two components $\mathcal{T}_{s}$ and $\mathcal{T}_{\text {es }}$, which sum to minus the depth-integrated Lagrangian Stokes drift, $\mathcal{T}_{s}^{L}$, and lie on opposite sides of a circle; $\mathcal{T}_{s}$ makes an angle $\theta$ to the north, where $\tan \theta=1 / 2 \delta_{e}^{2} / \delta_{s}^{2}$. The total water transport (Eulerian plus Lagrangian) then has components over two depth scales: $\mathcal{T}_{s}+\mathcal{T}_{s}^{L}$ over the Stokes depth; $\mathcal{T}_{\text {es }}+\mathcal{T}_{s}$ over the Ekman depth.

\section{b. Linearly varying eddy viscosity}

We expect the solutions obtained above with the constant viscosity to give the correct qualitative effects of the Coriolis-Stokes forcing. To substantiate this claim, particularly in the case that $\delta_{e} \gg \delta_{s}$, we briefly analyze here the solution obtained with an eddy viscosity that increases linearly with depth. This models the increased efficiency of turbulence to mix momentum farther from the boundary. Very near the surface, within the distance of a significant wave height, wave breaking and other processes significantly change the mixing and more sophisticated modeling is required (see Craig and Banner 1994). Below this layer, however a linear mixing length model is adequate according to Craig and Banner (1994). Hence we retain a simple model that contains just Ekman dynamics plus the Coriolis-Stokes forcing, but now we expect that the turbulent stress is better represented.

The eddy viscosity is now specified to be

$$
\kappa_{m}(z)=-\kappa v_{*} z=\kappa v_{*} z_{+},
$$

when $z<0 ; \kappa=0.4$ is the von Kármán constant, and $z_{+}=-z$. The momentum equations then reduce to

$$
\frac{\partial}{\partial \zeta}\left(\zeta \frac{\partial \mathcal{U}}{\partial \zeta}\right)-\zeta \mathcal{U}=\zeta u_{s}
$$

where $\zeta^{2}=i 4 f / \kappa v_{*} z_{+}{ }^{1}$ The particular solution is obtained by the method of variation of parameters, giving (following Madsen 1977; Lewis and Belcher 2004)

$$
\begin{aligned}
\mathcal{U}= & {\left[\frac{2 v_{*}}{\kappa}+4 i \int_{0}^{\hat{z}_{+}} I_{0}(\sqrt{8 i t}) \hat{u}_{s}(t) d t\right] K_{0}(\zeta) } \\
& +4 i I_{0}(\zeta) \int_{\hat{z}_{+}}^{\infty} K_{0}(\sqrt{8 i t}) \hat{u}_{s}(t) d t
\end{aligned}
$$

where $I_{0}$ and $K_{0}$ are modified Bessel functions (Abramowitz and Stegun 1972),

$\hat{z}_{+}=z_{+} / \delta_{e}, \delta_{e}=2 \kappa v_{*} / f, \quad$ and $\quad \hat{u}_{s}(t)=U_{s} \exp \left(-t \delta_{e} / \delta_{s}\right)$.

In the limits that $\delta_{e} / \delta_{s} \gg 1$ and $|z| \gg \delta_{s}$, the solution simplifies to

$$
\mathcal{U} \approx \frac{2 v_{*}}{\kappa}\left(1-i \frac{U_{s} \delta_{s}}{U_{e} \delta_{e}}\right) K_{0}(\zeta)=\left(1-i E_{s}\right) \mathcal{U}_{e},
$$

\footnotetext{
${ }^{1}$ Strictly speaking, there is another term representing the redistribution of momentum within the Stokes layer, which arises from the nonzero vertical gradient of eddy viscosity (Jenkins 1989). Since we are interested in the flow beneath this region, when $\delta_{e} / \delta_{s} \gg 1$, this is captured in the surface stress boundary condition.
} 
where $\mathcal{U}_{e}=2\left(v_{*} / \kappa\right) K_{0}(s)$ is the solution for the standard Ekman problem with no Coriolis-Stokes forcing. This is in agreement with what was found with the constanteddy-viscosity case. This agreement is no accident. In this limit of $\delta_{e} / \delta_{s} \gg 1$, the Stokes response [the last term in (18)] to the $\mathbf{f} \times \mathbf{u}_{s}$ forcing is negligible over most of the depth of the wind driven layer, as is the corresponding contribution to the net transport. Hence the waveinduced Eulerian transport, which is to equal $-\mathbf{T}_{s}$ by the integral constraint (6), must be carried by the Ekman-Stokes component of the solution. The significance is that, in this limit of $\delta_{e} / \delta_{s} \gg 1$, which is the limit appropriate for much of the ocean mixed layer, the Eulerian wave-driven transport is carried through the same depth as the wind-driven transport. We have shown here that this result follows through independently of the eddy-viscosity model used to compute the turbulent stress.

\section{c. Effective boundary condition for a shallow wave forcing}

Above it was shown how in the limit of $\delta_{e} / \delta_{s} \gg 1$, when the Ekman-Stokes layer is deep in comparison with the depth of the Coriolis-Stokes forcing, $\mathbf{f} \times \mathbf{u}_{s}$, the effect of the forcing on the current profile reduces to a canonical form. We now develop an argument to show how this can be understood as the Coriolis-Stokes forcing changing the boundary condition on the winddriven layer.

Recall that the Coriolis-Stokes forcing arises from a stress caused by the orbital motions associated with the surface wave [see (2)]. Hence the momentum equation governing the wind-driven layer can be written

$$
\rho f \hat{\mathbf{z}} \times \mathbf{u}=\frac{\partial \tau_{\text {tot }}}{\partial z}=\frac{\partial}{\partial z}\left(\tau+\tau_{w}\right),
$$

where the total stress, $\boldsymbol{\tau}_{\text {tot }}$ is the sum of the turbulent stress $\boldsymbol{\tau}$ and the wave-induced stress $\tau_{w}=-\rho \overline{\tilde{v} \tilde{w}}$, defined in Eq. (2). In the limit of $\delta_{e} / \delta_{s} \gg 1$, the Stokes layer is thin and below the Stokes layer the waveinduced stress is zero. Hence the total stress below the Stokes layer is just the turbulent stress. Now, a scale analysis of (20) shows that the vertical gradient of the total stress is small when $|z| \ll \delta_{e}$. Hence, as in the atmospheric boundary layer, the total stress within a very shallow layer is approximately constant with height. So the total stress just below the Stokes layer equals the total stress at the surface. At the surface the turbulent stress equals the applied wind stress, $\boldsymbol{\tau}_{0}$, and the wave-induced stress is given, on integrating (2), by

$$
\boldsymbol{\tau}_{\boldsymbol{w}}(0)=-\int_{-\infty}^{0} \rho \mathbf{f} \times \mathbf{u}_{\boldsymbol{s}} d z=-\boldsymbol{\rho} \mathbf{f} \times \mathbf{T}_{\boldsymbol{s}}
$$

Here $\mathbf{T}_{s}$ is the vector depth-integrated wave-driven transport. So the total stress just below the Stokes layer, which equals the turbulent stress just below the Stokes layer, is the applied wind stress plus the surface wave induced stress (21). Putting these two results together, the Ekman-Stokes layer can be modeled using standard Ekman balance

$$
\rho f \hat{\mathbf{z}} \times \mathbf{u}=\frac{\partial \boldsymbol{\tau}}{\partial z},
$$

subject to the effective boundary conditions

$$
\begin{aligned}
& \boldsymbol{\tau}= \boldsymbol{\tau}_{0}-\rho \mathbf{f} \times \mathbf{T}_{\boldsymbol{s}}=\rho v_{*}^{2}\left(\hat{\boldsymbol{\tau}}_{0}-\hat{\mathbf{z}} \times \hat{\mathbf{u}}_{s} E_{s}\right) \text { on } z=0, \\
& \mathbf{u} \rightarrow 0 \text { as } z \rightarrow-\infty
\end{aligned}
$$

for arbitrary wind and wave directions (with hats denoting unit vectors). The turbulent stress $\tau$ can then be modeled using any standard technique.

Figure 5 demonstrates use of the effective boundary condition. The figure shows, for these cases when $\delta_{e} / \delta_{s}$ $\approx 6$, good agreement between mean current profiles calculated with the resolved profile of Coriolis-Stokes forcing and with the effective boundary condition (which are plotted only below the Stokes depth). Also shown is the total Eulerian plus Lagrangian motion (which is the speed of movement of water parcels), obtained by adding the Stokes drift to the Eulerian solutions. The profiles show that, for these particular parameters, the speeds near the surface are then close to the standard Ekman solution, but deeper they follow the Stokes-Ekman form.

The derivation of the effective boundary condition shows that for the parameter regime of most interest in the ocean, it is the depth-integrated wave-induced transport that needs to be known to capture the effects of the Coriolis-Stokes forcing on the bulk of the flow. The detailed vertical profile of the Stokes drift is unimportant. This motivates the use of a single monochromatic wave in LES and the comparisons with observations.

This finding also has implications for representation of the Coriolis-Stokes term in ocean general circulation models. These models do not typically have sufficient vertical resolution to compute the flow within the upper part of the mixed layer where the Coriolis-Stokes force acts. The present analysis shows that the effect of this forcing on the mixed layer can be represented by changing the boundary condition on the standard Ekman equations.

\section{Large-eddy simulation of the Ekman-Stokes layer}

We have reappraised simple models for the winddriven mixed layer that show how the Coriolis-Stokes force changes the mean current profile through all depths. These models represented the turbulent stress associated with three-dimensional overturning turbulent motions through simple eddy-viscosity models. These turbulent motions are represented more faith- 
$U \mathrm{~s}=0.017 \mathrm{~m} / \mathrm{s}$

$\mathrm{Us}=0.034 \mathrm{~m} / \mathrm{s}$

Us $=0.068 \mathrm{~m} / \mathrm{s}$

Us $=0.271 \mathrm{~m} / \mathrm{s}$
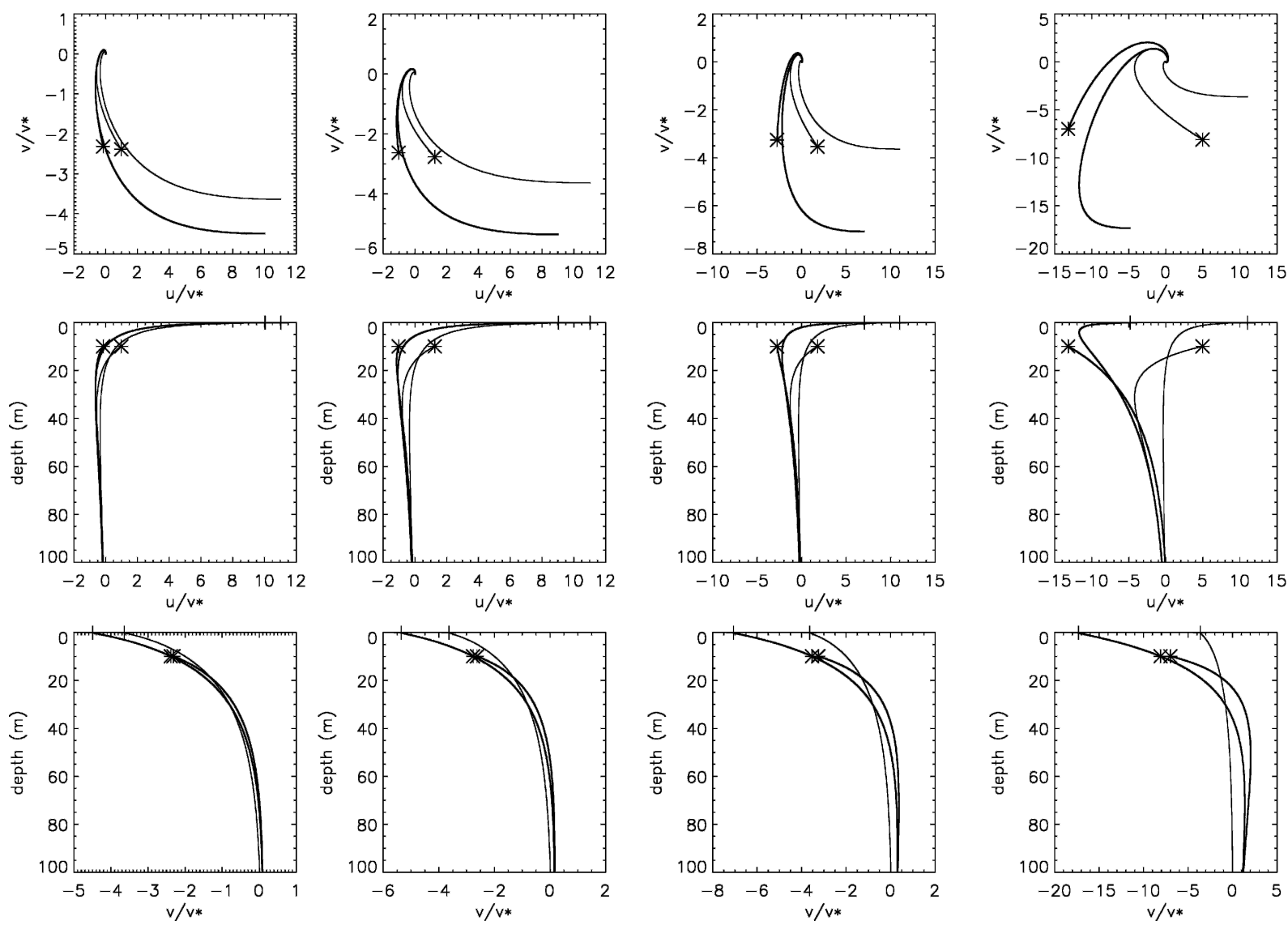

FIG. 5. Mean current profiles calculated with the effective boundary condition, with turbulence parameterized with a linear eddyviscosity model. There are four values of $U_{s}$, and $v_{*}=6.1 \times 10^{-3} \mathrm{~m} \mathrm{~s}^{-1}$ and $f=10^{-4} \mathrm{~s}^{-1}$, so that $\delta_{e}=v_{*} / f \approx 61 \mathrm{~m}$ and $k=0.0525 \mathrm{~m}{ }^{-1}$, so that $\delta_{s} \approx 10 \mathrm{~m}$. Hence $\delta_{e} / \delta_{s} \approx 6 \gg 1$. (top) Hodographs, (middle) vertical profile of $u / v_{*}$, and (bottom) vertical profile of $v / v_{*}$ : thick lines, full solution with Coriolis-Stokes forcing; thin lines, standard Ekman solution; thick line with star, solution with the effective boundary condition; thin line with star, total Eulerian plus Lagrangian drift. Solutions with effective boundary condition plotted from $z=-\delta_{s}$ downward.

fully in this section through large-eddy simulation (hereinafter LES), where the fully nonlinear equations of motion are integrated forward in time with sufficient resolution to compute explicitly the large-scale turbulent motions. Only the small-scale turbulence is parameterized. In this section the mean current profiles are computed by an LES model of the turbulent EkmanStokes layer.

Following Skyllingstad and Denbo (1995) and McWilliams et al. (1997), we perform LES of the wavefiltered Craik-Leibovich equations to account for wavelength-averaged effects of surface waves. With this procedure the momentum equation becomes

$$
\frac{D \mathbf{u}}{D t}+\mathbf{f} \times\left(\mathbf{u}+\mathbf{u}_{s}\right)=-\nabla \pi+\mathbf{u}_{\mathrm{s}} \times \boldsymbol{\omega}+\mathrm{SGS} .
$$

Here $\mathbf{u}$ is the wave-averaged Eulerian velocity, $\mathbf{f}=f \hat{\mathbf{z}}$ is the Coriolis parameter, $\hat{\mathbf{z}}$ is the upward unit vector, $\boldsymbol{\omega}=$ $\nabla \times \mathbf{u}$ is the local vorticity vector, and $D / D t=\partial / \partial t+$ $\mathbf{u} \cdot \boldsymbol{\nabla}$ is the material derivative. The subgrid-scale processes (denoted SGS) are parameterized using a standard Smagorinsky model. Last, $\pi$ is the generalized pressure given by

$$
\pi=\frac{p}{\rho_{0}}+\frac{1}{2}\left(\left|\mathbf{u}+\mathbf{u}_{\mathrm{s}}\right|^{2}-|\mathbf{u}|^{2}\right) .
$$

We consider the simplest problem that highlights clearly the role of wave-induced processes when the density is prescribed to be constant with depth and a single monochromatic wave. (Note that this assumption of monochromatic waves is not likely to be important: the analysis above shows how it is the depth-integrated wave-induced transport that is important, not the vertical variation of Stokes drift.)

The governing equations then contain the CoriolisStokes forcing, $\mathbf{f} \times \mathbf{u}_{s}$, and also the vortex force, $\mathbf{u}_{s} \times \boldsymbol{\omega}$, 
which represents the straining of the vorticity associated with resolved mean and turbulent motions by the Stokes drift. This latter term gives rise to Langmuir circulations (as reviewed by Leibovich 1983), which lead to enhanced vertical mixing. The LES with the vortex force yields a turbulent boundary layer with elongated Langmuir vortices on a range of scales, whose dynamics are described in Skyllingstad and Denbo (1995), McWilliams et al. (1997), and Teixeira and Belcher (2002). Here we focus on the mean current profiles produced by the LES.

The equations are integrated numerically using a code based on the atmospheric boundary layer code BLASIUS (Wood and Mason 1993), which has been modified to include the two wave-forcing terms. The code is run in LES mode (Brown et al. 2001) using a Smagorinsky subgrid model. For further model details refer to Wood et al. (1998). The domain is periodic and isotropic in the horizontal directions spanning $120 \mathrm{~m}$ with a resolution of $3 \mathrm{~m}$. In the vertical direction 200 grid points span $90 \mathrm{~m}$ with a resolution of $0.46 \mathrm{~m}$. This is similar to the $3 \mathrm{~m} \times 3 \mathrm{~m} \times 0.6 \mathrm{~m}$ resolution used by McWilliams et al. (1997), which is vertically uniform. Our simulations also have uniform vertical resolution except in the upper $1 \mathrm{~m}$ where we use a stretched grid over four levels. The most significant difference between our simulations and those of McWilliams et al. (1997) is in the stratification. In the McWilliams et al. (1997) study, the upper $33 \mathrm{~m}$ is neutrally buoyant and the rest is stably stratified. Here we simplify and make the whole domain neutrally buoyant to isolate clearly the effects of the wave processes. At the surface a constant wind stress is applied in the $x$ direction such that at $z=-z_{0}$,

$$
\kappa_{m} \frac{\partial u}{\partial z}=v_{*}^{2} \quad \text { and } \quad \kappa_{m} \frac{\partial v}{\partial z}=0,
$$

where $\kappa_{m}$ is the mixing-length eddy viscosity, which parameterizes the stress very near the surface, $v_{*}$ is the friction velocity in the water, and $z_{0}$ is the roughness length. No attempt is made to represent mixing by breaking waves. At the lower boundary, $z=-90 \mathrm{~m}$, a no-slip condition is imposed (although the domain was sufficiently deep that this boundary condition did not play a dynamical role). The code was checked by performing a simulation with the parameters of McWilliams et al. (1997). Vertical profiles of the mean flow and turbulence statistics from our simulation (not shown) are in good agreement with McWilliams et al. (1997).

A total of 16 simulations have been performed for a range of $k$ and $U_{s}$ wave parameters. Each of the simulations had $v_{*}=6.1 \times 10^{-3} \mathrm{~m} \mathrm{~s}^{-1}$ (corresponding to a $10-\mathrm{m}$ atmospheric wind speed, $U_{10} \approx 5 \mathrm{~m} \mathrm{~s}^{-1}$ ) and $f=$ $1 \times 10^{-4} \mathrm{~s}^{-1}$. The roughness of the sea surface from below is taken to be $z_{0}=0.1 \mathrm{~m}$, which is smaller than the values used by Craig and Banner (1994), but expe- rience with the linear eddy-viscosity model indicates that the current profiles in the bulk of the Ekman layer are insensitive to this parameter. The wave parameters are $k=0.02625,0.0525,0.105$, and $0.210 \mathrm{~m}^{-1}$, which yields wavelength $\lambda=240,120,60$, and $30 \mathrm{~m}$ and a surface Stokes drift of $U_{s}=0,0.017,0.034,0.068$, and $0.271 \mathrm{~m} \mathrm{~s}^{-1}$. Lewis and Belcher (2004) show that, with a constant viscosity, the transient motions, with and without Coriolis-Stokes forcing, decay on the inertial period $T=O(2 \tau / f) \approx 6 \times 10^{4} \mathrm{~s}$. Hence each run was integrated to $2 \times 10^{5} \mathrm{~s}$, which is a little over three inertial periods. Profiles of the second-order turbulence moments were monitored and satisfactory steady state was seen after this time. Mean flow and turbulent statistics were gathered starting at $5000 \mathrm{~s}$ and computed from instantaneous horizontal averages that are taken approximately every $10 \mathrm{~s}$.

\section{LES results and comparison with eddy-viscosity closure model}

The mean current profiles obtained from the LES are now compared with the simple eddy-viscosity closure model discussed in section $2 \mathrm{~b}$. Figure 6 shows solutions from four simulations, all with $k=0.0525 \mathrm{~m}^{-1}(\lambda=120$ $\mathrm{m})$ but with increasing wave amplitudes, and hence increasing $U_{s}$. [Since $E_{s} \propto U_{s} /(2 k)$, increasing $k$ has the same qualitative effect as decreasing $U_{s}$.]

The upper panels show hodographs of the locus of the current vector as the depth increases. The middle panels show corresponding profiles of mean along-wind velocity, $\bar{u}$ against depth. The lower panels show the corresponding mean across-wind velocity, $\bar{v}$, against depth. In each panel the thicker solid line is the LES data, the thinner solid line is the solution from the model with linearly varying eddy-viscosity closure, and the dashed line is LES data from a run without wave forcing (that is the pure Ekman solution).

First consider the LES solutions. Even for moderate values of the Stokes drift, for example when $a=0.95 \mathrm{~m}$ so that $a k=0.05, U_{s}=0.034 \mathrm{~m} \mathrm{~s}^{-1}$ and $E_{s}=0.9$, the LES with wave forcing is markedly different from the pure Ekman solution without wave forcing. The effect of the Coriolis-Stokes force is primarily to rotate the current profiles southward, consistent with the effective boundary condition ideas in section 2 .

Comparing the LES solutions with the solution from the model with linearly increasing eddy viscosity we see that there is a reasonably good quantitative agreement, particularly within the bulk of the Ekman-Stokes layer. Very close to the surface, within the layer affected directly by the Coriolis-Stokes forcing, $|z|<\delta_{s}$ $\approx 10 \mathrm{~m}$, the LES shows less shear than the solution from the closure model. It seems likely that the enhanced mixing due to the Langmuir circulations, which are undoubtedly present in the LES, reduce the shear there. The eddy-viscosity model has a prescribed linearly varying eddy viscosity, which makes no attempt to represent these Langmuir circulations. Nevertheless, 

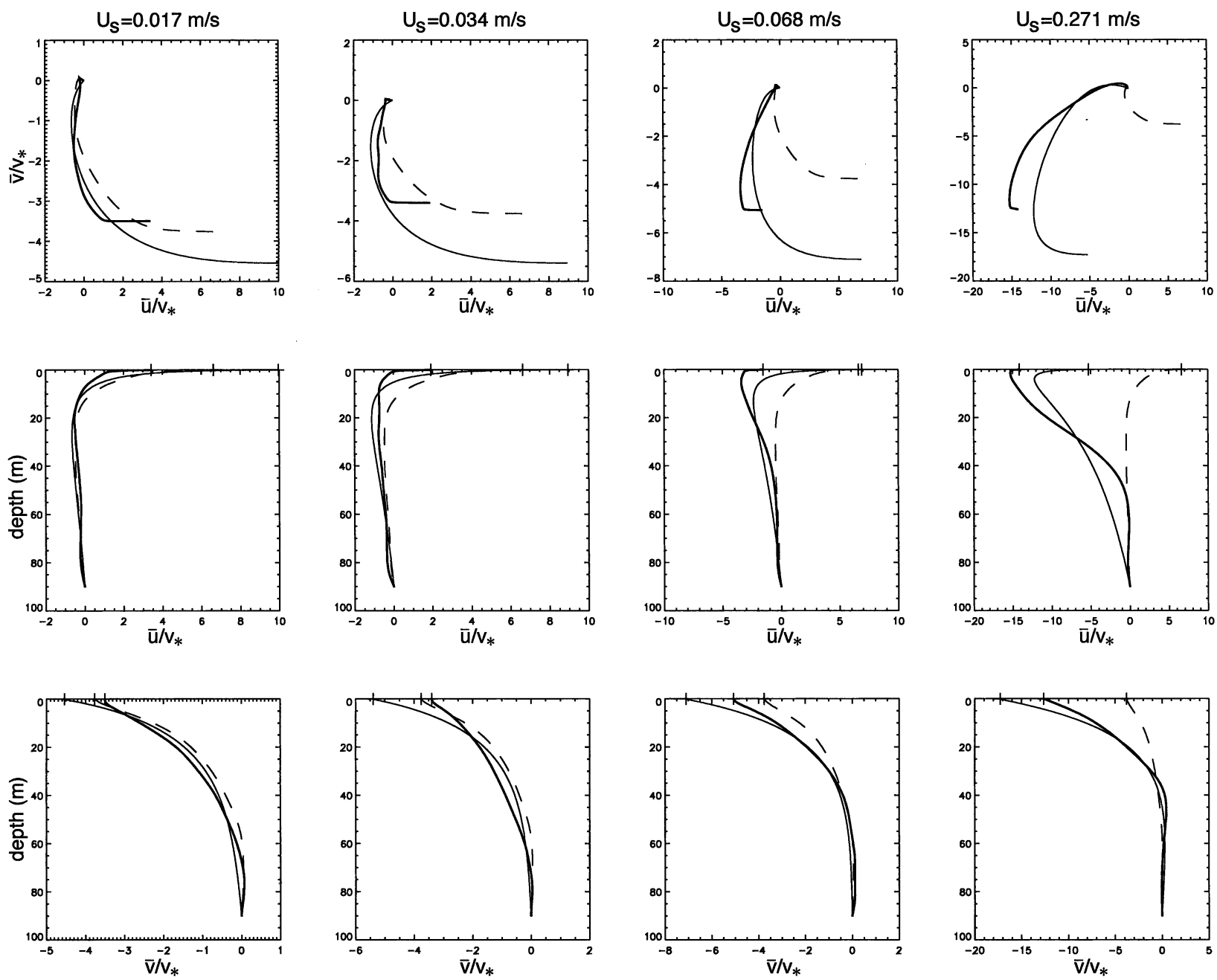

FIG. 6. Plots comparing LES solutions with wave forcing (thick solid lines) with LES solutions without wave forcing (thin dashed solid lines) and linear eddy-viscosity closure model solutions (thin solid lines) for a range of $U_{s}$ and $k=0.0525 \mathrm{~m}^{-1}$ : (top) hodographs of $\bar{v} / v_{*}$ against $\bar{u} / v_{*} ;$ (middle) vertical profile of $\bar{u} / v_{*}$ and (bottom) vertical profile of $\bar{v} / v_{*}$. The eddy-viscosity closure model solution is in remarkably good qualitative agreement with the wave-forced LES solution. The wind forcing and wave propagation are both directed along the positive $x$ axis. With varying $U_{s}$, the corresponding wave amplitude $a$ and $E_{s}$ are $U_{s}=0.017 \mathrm{~m} \mathrm{~s}^{-1}\left(a=0.67 \mathrm{~m}, E_{s}=0.4\right)$, $U_{s}=0.034 \mathrm{~m} \mathrm{~s}^{-1}\left(a=0.95 \mathrm{~m}, E_{s}=0.9\right), U_{s}=0.068 \mathrm{~m} \mathrm{~s}^{-1}\left(a=1.34 \mathrm{~m}, E_{s}=1.7\right)$, and $U_{s}=0.271 \mathrm{~m} \mathrm{~s}^{-1}\left(a=2.68 \mathrm{~m}, E_{s}=6.9\right)$.

over the bulk of the layer the simple model does a reasonable job. We conclude that the Coriolis-Stokes forcing can significantly change the mean current profile and that the effects on the bulk of the wind driven layer can be captured with even a simple turbulence model.

\section{Evidence for effects of Coriolis-Stokes forcing in observational data}

In this section we seek evidence of the effects of the Coriolis-Stokes forcing in observed wind-driven current profiles. Extracting the mean wind-driven current profile from the background of wave orbital motions, inertial oscillations, and geostrophic eddies requires so- phisticated and sensitive instruments that can be deployed for long periods. Consequently it is only relatively recently that data have been collected that can be compared with models of the wind-driven current profile, and there are relatively few such data. Here we use data described in Price and Sundermeyer (1999), namely the LOTUS data and the EBC data. The profiles show considerable deviations from the classical Ekman profiles, which Price and Sundermeyer (1999) attribute to dynamical effects of mixed layer stratification and diurnal variations in the mixed layer depth. However, Lewis and Belcher (2004) point out several potential problems with this interpretation. First, Price and Sundermeyer (1999) use a laminar model to represent the turbulent ocean boundary layer and so do 
not obtain the logarithmic velocity profile observed close to the surface below the breaking wave zone (Terray et al. 1996). Second, their solutions, while matching the observed angle of deflection of the subsurface current, give surface current deflections smaller than the values cited in Huang (1979). Hence we examine whether a model that incorporates Coriolis-Stokes forcing, but with uniform density, can explain the deviations from classical Ekman profiles.

The Long-Term Upper-Ocean Study (LOTUS3) data (Briscoe and Weller 1984; Price et al. 1987) were collected in the western Sargasso Sea $\left(34^{\circ} \mathrm{N}, 70^{\circ} \mathrm{W}\right)$ spanning 160 days during the summer months of 1982. The current profile was recorded by vector-measuring current meters mounted on from $\mathrm{R} / \mathrm{P} F L I P$, which is a stable platform and so does not suffer contamination from mooring motions. The data were processed by first subtracting the geostrophic velocity, taken to be the velocity at some depth, in this case $50 \mathrm{~m}$ which is deeper than the wind penetration depth. Then, since the wind direction was not steady over the 160-day period, daily averages of wind and current were rotated such that the wind was aligned with a nominal north. These daily data were then averaged over the 160 days. Further analysis details are given in Price and Sundermeyer (1999) and references therein.

The eastern boundary current (EBC) data are reported in Chereskin (1995). This dataset is taken from a mooring $400 \mathrm{~km}$ off the coast of North California $\left(37^{\circ} \mathrm{N}, 128^{\circ} \mathrm{W}\right)$ and was collected over a 6-month period from 8 April to 20 October 1993 using ADCP and buoy wind observations. The data required no rotating prior to averaging as the wind was sufficiently unidirectional over the 6-month collecting period. Chereskin (1995) and the references therein describe how the sampling strategy of the ADCP and the subsequent analysis of the data were designed to minimize effects of mooring motions.

Price and Sundermeyer (1999) also describe a third dataset, the Trans-Pacific Hydrographic Section (TPHS) data, which was originally reported in Wijffels et al. (1994). The Coriolis parameter at this location is much smaller and the corresponding Ekman depth scale is larger (of order $150 \mathrm{~m}$ ) and the assumption of uniform density ceases to be reliable (Lewis and Belcher 2004), and so we consider this dataset no further.

Both the LOTUS and the EBC data are then good data to compare with the model: they both measured Eulerian currents and were as free from contamination from moorings as is possible.

\section{a. Determination of the surface wave parameters}

Neither of the datasets used here measured the surface waves. Here we use two methods to estimate the wave parameters.

First, the wave parameters are determined assuming a fully developed sea. Thus the Pierson and Moskowitz
(1964) wave spectrum ${ }^{2}$ was used to deduce the wave parameters from the measured wind speed. The Peirson-Moskowitz spectrum is integrated over frequency to calculate the surface Stokes drift and the peak wavenumber of the spectrum is used for the exponential depth scale.

Second, we estimate parameters of the surface waves from the European Centre for Medium-Range Weather Forcasts (ECMWF) reanalysis, which contains global analysis of the wave properties. In this system wave spectra are computed from the ECMWF integrated forecasting system, which includes the WAM wave model, with some surface wave measurements assimilated (e.g., Janssen et al. 1997). In the wave data the magnitude and direction of the depth-integrated wave-induced transport are calculated from 6 hourly data dumps. For comparison with the LOTUS3 data, the directional information is discarded as the LOTUS3 current data are realigned with a nominal north each day. For the EBC data there is no such adjustment, and so the wave transports are projected onto the mean wave direction. Figure 7 shows values of the depthintegrated wave-induced transport obtained from the ECMWF data together with comparisons with values estimated from the Pierson-Moskowitz spectrum. The top row is for the LOTUS3 campaign and the bottom row is for the EBC campaign. The left-hand plots show maps of the depth-integrated wave-driven transport averaged over the observation period. The location of the LOTUS3 $\left(34^{\circ} \mathrm{N}, 70^{\circ} \mathrm{W}\right)$ and $\mathrm{EBC}\left(37^{\circ} \mathrm{N}, 128^{\circ} \mathrm{W}\right)$ sites are marked with boxes. The right-hand plots show time series of the transport (solid line) of the depth-integrated wave-driven transport at the observation points. Also shown is the value obtained from the Pierson-Moskowitz spectrum with the time-averaged wind speed, which shows encouraging agreement with the average obtained from the full ECMWF data.

In section 2 we showed that, provided the depthintegrated transport is correct, the detailed profile of Stokes drift is unimportant in determining the current profile. Hence, in the model profiles shown below the waves are represented as monochromatic, but with parameters $U_{s}$ and $k$ chosen such that the depth-integrated transport is equal to the value from the ECMWF reanalysis. Either $U_{s}$, the surface value of the Stokes drift, or $k$ needs to be specified. Here we show results with $U_{s}$ determined from the value obtained from the Peirson-Moskowitz spectrum at the mean wind speed, and then $k$ inferred to yield the correct depth-integrated transport. Very similar current profiles were obtained by taking $k$ from the peak in the Peirson-Mosko-

\footnotetext{
${ }^{2}$ The $10-\mathrm{m}$ wind speed from the observations are scaled to $19.5-\mathrm{m}$ wind speed [which is the independent parameter in the Pierson and Moskowitz (1964) spectrum] using the method described in section 1 .
} 

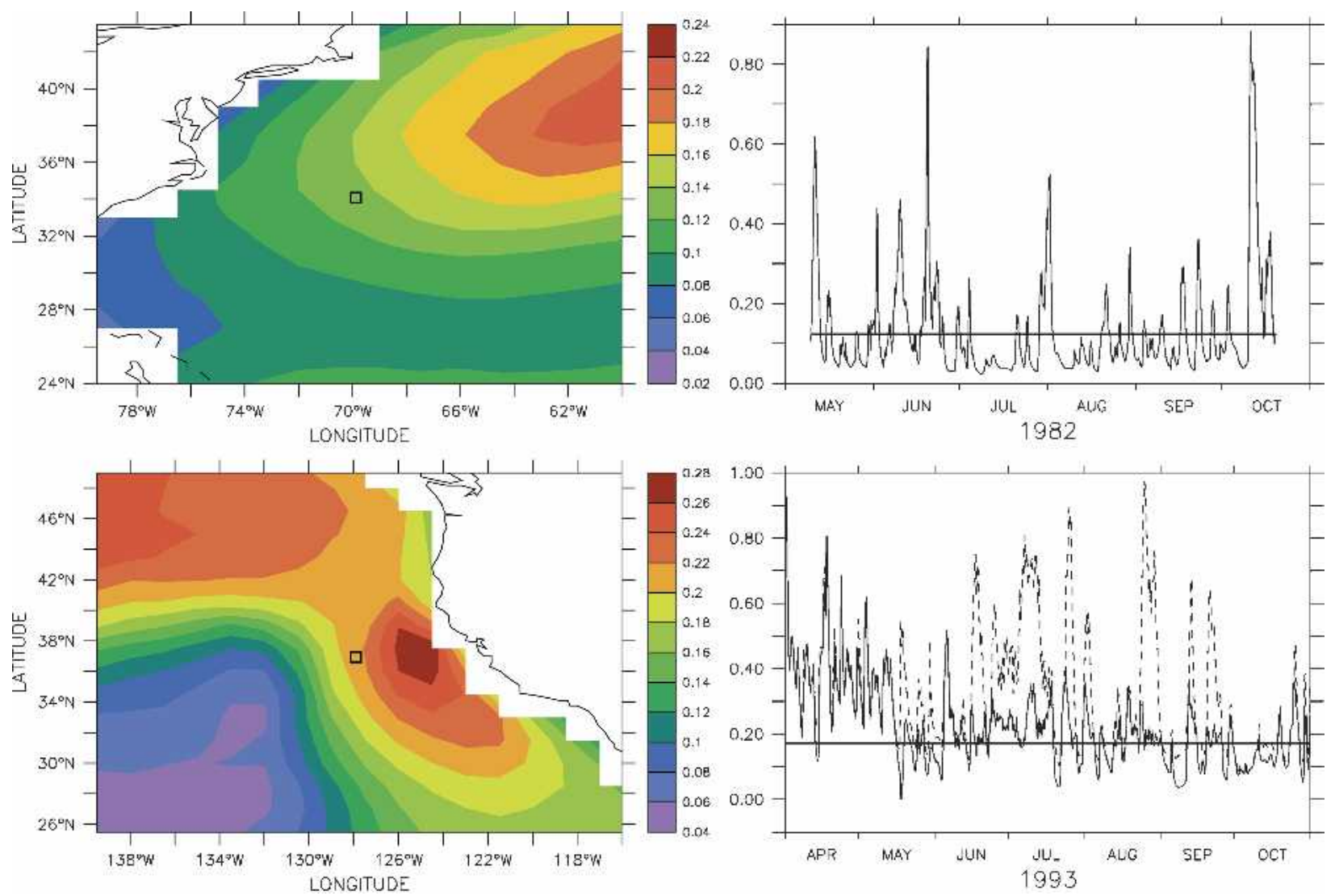

FIG. 7. Depth-integrated wave-driven transport obtained from the ECMWF reanalysis data $\left(\mathrm{m}^{2} \mathrm{~s}^{-1}\right)$ : (top) LOTUS3 data and (bottom) EBC data. (left) Maps of depth-integrated wave-driven transports averaged over the observation periods. (right) Time series of the depth-integrated wave-driven transport at the measurement locations. Also shown is the value calculated from the PeirsonMoskowitz spectrum with the average wind speed. Since the EBC data were interpreted as if the mean wind direction were constant, the instantaneous wave-induced transport is projected into the mean direction (the dashed line in the bottom right is the magnitude of the vector transport).

witz spectrum and then inferring $U_{s}$ to yield the correct transport.

\section{b. Comparison with the observations}

Figure 8 shows the observed current profiles. Also shown are profiles from the simple model with the turbulent stress modeled with a linear eddy viscosity, both with and without the Coriolis-Stokes. The simple model solutions are calculated for an infinite layer of uniform density and then postprocessed in the same way as the observations. Thus the profile is "rezeroed" by subtracting the velocity at the depth used by Price and Sundermeyer (1999) as the depth of the geostrophic, namely $50 \mathrm{~m}$ for the LOTUS3 data and $60 \mathrm{~m}$ for the EBC data.

The solutions from the simple model that account for the Coriolis-Stokes force with wave properties deduced assuming a fully developed sea (the solid line) shows markedly improved agreement with the data when compared with the classical Ekman solution, obtained when the Coriolis-Stokes force is set to zero (the dotdashed line). Also shown is the range that the theory can take when the wave parameters are varied. The solid shading denotes the range of solutions from the model when the wavelength of the waves is changed by $\pm 50 \%$. The hatched envelope arises from changing the square of the wave amplitude, and hence $U_{s}$, by $\pm 50 \%$. In general, the observational data lie within the bounds of uncertainty, although the data appear to be rotated even more than the model. Accounting for CoriolisStokes forcing certainly improves the agreement with the classical Ekman solution, which lies some distance away from the data.

The dashed line in Fig. 8 denotes the solution from the simple model when the wave parameters are calculated using the depth-integrated transport from the ECMWF data. The hodograph is turned slightly less than from the fully developed sea because the depthintegrated transport is smaller than for a fully developed sea. We note that, as shown in Fig. 7, there is spatial variability in the ECMWF estimates for the depth-integrated transport, and so it is possible that the actual transport was higher than estimated, thereby bringing the two model curves together. 

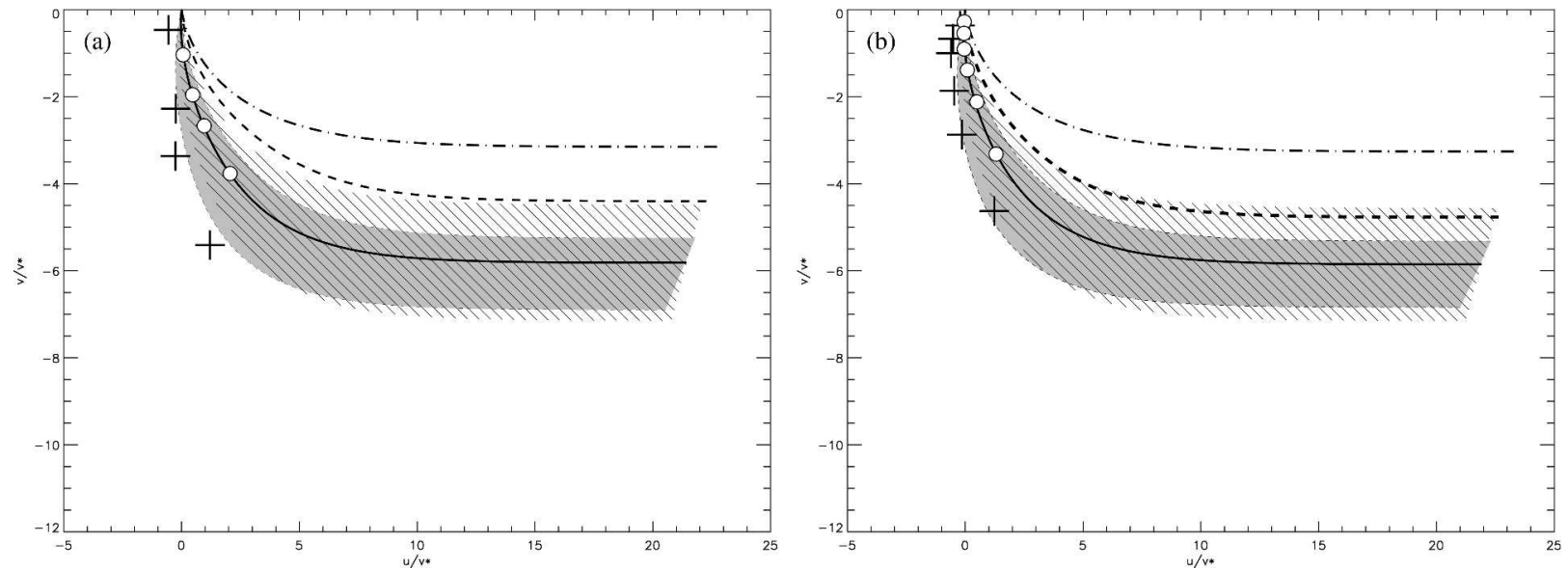

FIG. 8. Hodograph comparisons between simple analytic model (assuming a fully developed sea) and observational measurements from (a) LOTUS3 $\left(v_{*}=8.3 \times 10^{-3} \mathrm{~m} \mathrm{~s}^{-1}, z_{0}=1.6 \times 10^{-3} \mathrm{~m}\right)$ and (b) EBC $\left(v_{*}=9.4 \times 10^{-3} \mathrm{~m} \mathrm{~s}^{-1}, z_{0}=1.4 \times 10^{-3} \mathrm{~m}\right)$ datasets. Dot-dash line is the model with $u_{s}=0$-no wave effects. Heavy solid line is the model with wave effects. Solid shaded envelope is $k$ (from FDS) $\pm 50 \%$. Hatched envelope is $U_{s}^{2}$ (i.e., $a^{2}$ from FDS) $\pm 50 \%$. Heavy dashed line is hodographs using ECMWF wave model values for wave-induced transport. Crosses denote observational measurements, and white dots represent corresponding depths in analytical model for (a) 5, 10, 15, and $25 \mathrm{~m}$ and (b) 8, 16, 24, 32, 40, and $48 \mathrm{~m}$.

We conclude that the changes to the current profile due to Coriolis-Stokes forcing are consistent with the differences between observations and the classical Ekman profile. The comparisons given here indicate that there is some quantitative agreement between the observations and the simple model once account has been made of the uncertainties in the wave parameters, although there is a suggestion that the measured hodographs are rotated even further.

\section{Concluding remarks}

We have examined the role of the Coriolis-Stokes forcing, $\mathbf{f} \times \mathbf{u}_{s}$, in shaping the mean current profile in the wind-driven ocean mixed layer. At first sight this force might be thought to be small since it involves the Stokes drift, which scales on the wave slope squared. Estimates show that in conditions of even modest sea state the depth-integrated transport associated with this forcing can be a considerable fraction of the depthintegrated wind-driven transport. This observation motivated the present more detailed examination of the role of this forcing.

Simple analytical solutions, based on parameterizing the turbulence using simple eddy viscosities, show how the Coriolis-Stokes forcing interacts with the Coriolis force and the turbulent stress divergence. The resulting wind-driven current profile is characterized by two length scales, namely the depth scale of the wind-driven layer, $\delta_{e}$, and the depth of penetration of the CoriolisStokes forcing, $\delta_{s}$. In typical ocean conditions $\delta_{s} \ll \delta_{e}$, and yet the current profile is completely changed through all depths, with the current vectors rotated farther away from the direction of the wind stress. The reason is that the Coriolis-Stokes forcing absorbs a fraction of the applied wind stress, thus changing the effective boundary condition on the standard winddriven Ekman solution. A corollary to this finding is that the effects of the Coriolis-Stokes forcing can be represented simply by integrating the standard equations of motion, but with the boundary condition changed by an amount that depends on the depthintegrated Stokes drift. Hence there is no need for numerical ocean models to resolve explicitly the region affected directly by the Coriolis-Stokes forcing.

We investigated the relevance of these findings to the real ocean by comparing the results of the simple models to large-eddy simulations and observations. The LES resolve the large-scale turbulent motions, but represent the effects of the waves through their wavelength-averaged effects only. Nevertheless, the wind and wave conditions are prescribed and remain constant, giving clean data to compare with the simple theory. The observational data, taken from the LOTUS3 and EBC campaigns, on the other hand, contain the complexity of the real world, including variable wind speed and direction. Wave properties were not measured during the observations and so were estimated here by (i) assuming that the waves were fully developed with respect to the local wind speed and (ii) using ECMWF reanalysis data. When compared with both the LES and the observations the simple models that account for the Coriolis-Stokes forcing have shown encouraging agreement. This provides perhaps the first evidence of the signature of the CoriolisStokes forcing in observations. These findings suggest that future observations of the wind-driven mixed layer also need to measure surface wave properties.

Ultimately it is the wind that provides the momentum flux to the surface wind stress, with its wind-driven 
flow, and to the surface waves, with their associated the Coriolis-Stokes forcing. In the present paper the wind and waves have been specified separately. An important topic for future research will therefore be to examine the partition of the momentum flux between these two components.

Acknowledgments. We gratefully acknowledge the suggestions of the anonymous reviewers. The research presented in this paper was funded by the Leverhulme Trust under Grant F/00239/A and the U.S. Office of Naval Research under Grant N-00014-01-1-0133.

\section{REFERENCES}

Abramowitz, M., and I. A. Stegun, 1972: Handbook of Mathematical Function. Dover, 1046 pp.

Briscoe, M. G., and R. A. Weller, 1984: Preliminary results from the Long-Term Upper-Ocean Study (LOTUS). Dyn. Atmos. Oceans, 8, 243-265.

Brown, A. R., J. M. Hobson, and N. Wood, 2001: Large-eddy simulation of neutral turbulent flow over rough sinusoidal ridges. Bound.-Layer Meteor., 98, 411-441.

Chereskin, T. K., 1995: Direct evidence for an Ekman balance in the California Current. J. Geophys. Res., 100, 18 261-18 269.

Craig, P. D., and M. L. Banner, 1994: Modeling wave-enhanced turbulence in the ocean surface layer. J. Phys. Oceanogr., 24, 2546-2559.

Garratt, J. R., 1992: The Atmospheric Boundary Layer. Cambridge University Press, $316 \mathrm{pp}$.

Gnanadesikan, A., and R. A. Weller, 1995: Structure and instability of the Ekman spiral in the presence of surface gravity waves. J. Phys. Oceanogr., 25, 3148-3171.

Hasselmann, K., 1970: Wave-driven inertial oscillations. Geophys. Fluid Dyn., 1, 463-502.

Huang, N. E., 1979: On surface drift currents in the ocean. J. Fluid Mech., 91, 191-208.

Janssen, P., B. Hansen, and J. Bidlot, 1997: Verification of the ECMWF wave forecasting system against buoy and altimeter data. Wea. Forecasting, 12, 763-784.

Jenkins, A. D., 1986: A theory for steady and variable wind- and wave-induced currents. J. Phys. Oceanogr., 16, 1370-1377.

_ 1987: Wind and wave induced currents in a rotating sea with depth-varying eddy viscosity. J. Phys. Oceanogr., 17, 938-951.

_ 1989: The use of a wave prediction model for driving a near-surface current model. Dtsch. Hydrogr. Z., 42, 133-149.

Kenyon, K. E., 1969: Stokes drift for random gravity waves. $J$. Geophys. Res., 74, 6991-6994.
Leibovich, S., 1983: The form and dynamics of Langmuir circulations. Annu. Rev. Fluid Mech., 15, 391-427.

Lewis, D. M., and S. E. Belcher, 2004: Time-dependent, coupled, Ekman boundary layer solutions incorporating Stokes drift. Dyn. Atmos. Oceans, 37, 313-351.

Madsen, O. S., 1977: A realistic model of the wind-induced Ekman boundary layer. J. Phys. Oceanogr., 7, 248-255.

, 1978: Mass transport in deep-water waves. J. Phys. Oceanogr., 8, 1009-1015.

McWilliams, J. C., and J. M. Restrepo, 1999: The wave-driven ocean circulation. J. Phys. Oceanogr., 29, 2523-2540.

_ P. P. Sullivan, and C.-H. Moeng, 1997: Langmuir turbulence in the ocean. J. Fluid Mech., 334, 1-30.

Phillips, O. M., 1977: Dynamics of the Upper Ocean. Cambridge University Press, $336 \mathrm{pp}$

Pierson, W. J., and L. Moskowitz, 1964: A proposed spectral form for fully developed wind seas based on the similarity theory of S. A. Kitaigorodskii. J. Geophys. Res., 69, 5181-5203.

Pollard, R. T., 1970: Surface waves with rotation: An exact solution. J. Geophys. Res., 75, 5895-5898.

Price, J. F., and M. A. Sundermeyer, 1999: Stratified Ekman layers. J. Geophys. Res., 104, 20 467-20 494.

_, R. A. Weller, and R. R. Schudlich, 1987: Wind-driven ocean currents and Ekman transport. Science, 238, 1534-1538.

Skyllingstad, E. D., and D. W. Denbo, 1995: An ocean large-eddy simulation of Langmuir circulations and convection in the surface mixed layer. J. Geophys. Res., 100, 8501-8522.

Teixeira, M. A. C., and S. E. Belcher, 2002: On the distortion of turbulence by a progressive surface wave. J. Fluid Mech., $\mathbf{4 5 8}$ 229-267.

Terray, E. A., M. Donelan, Y. Agrawal, W. Drennan, K. Kahma, A. J. Williams III, P. Hwang, and S. Kitaigorodskii, 1996: Estimates of kinetic energy dissipation under breaking waves. J. Phys. Oceanogr., 26, 792-807.

Ursell, F., 1950: On the theoretical form of ocean swell on a rotating earth. Mon. Not. Roy. Astron. Soc. Geophys. Suppl., $6,1-8$.

Weber, J. E., 1983a: Attenuated wave-induced drift in a viscous rotating ocean. J. Fluid Mech., 137, 115-129.

, 1983b: Steady wind- and wave-induced currents in the open ocean. J. Phys. Oceanogr., 13, 524-530.

Wijffels, S., E. Firing, and H. Bryden, 1994: Direct observations of the Ekman balance at $10^{\circ} \mathrm{N}$ in the Pacific. J. Phys. Oceanogr., 24, 1666-1679.

Wood, N., and P. Mason, 1993: The pressure force induced by neutral, turbulent flow over hills. Quart. J. Roy. Meteor. Soc., 119, $1233-1267$.

, F. Hewer, and M. Hobson, 1998: BLASIUS Version 3.03. Documentation of a Model of Flow over Hills. Met Office, 71 $\mathrm{pp}$

Xu, Z., and A. J. Bowen, 1994: Wave- and wind-driven flow in water of finite depth. J. Phys. Oceanogr., 24, 1850-1866. 